\title{
Entire solutions of certain class of differential-difference equations
}

\author{
Fengrong Zhang ${ }^{1}$, Nana Liu', Weiran Lü ${ }^{1 *}$ and Chungchun Yang ${ }^{2}$
}

\author{
Correspondence: luwr@upc.edu.cn \\ ${ }^{1}$ College of Science, China \\ University of Petroleum (East China), \\ Qingdao, 266580, P.R. China \\ Full list of author information is \\ available at the end of the article
}

\begin{abstract}
As a continuation of our previous studies Liu et al. (J. Inequal. Appl. 2014:63, 2014), we will discuss the transcendental entire solutions of the following type of differential-difference equation: $f^{3}(z)+P_{1}\left(z, \Delta f, \ldots, f^{\prime}, \ldots, f^{(k)}\right)=\lambda_{1} e^{\alpha_{1} z}+\lambda_{2} e^{\alpha_{2} z}$, where $P_{1}$ is a linear polynomial in $f, \Delta f, \ldots, f^{(k)}$, with polynomials as its coefficients, and $\lambda_{1}, \lambda_{2}, \alpha_{1}, \alpha_{2} \in \mathbb{C}$ are nonzero constants such that $\alpha_{1} \neq \alpha_{2}$.
\end{abstract}

MSC: 30D35; 30A06

Keywords: transcendental entire solution; differential-difference equation; Nevanlinna theory

\section{Introduction}

In this paper, we shall adopt the standard notations in Nevanlinna's value distribution theory of meromorphic functions. For example, the characteristic function $T(r, f)$, the counting function of the poles $N(r, f)$, and the proximity function $m(r, f)$ (see, e.g., $[1,2]$ ).

Let $f$ be a meromorphic function. Recall that $\alpha \neq \equiv 0, \infty$ is a small function with respect to $f$, if $T(r, \alpha)=S(r, f)$, where $S(r, f)$ denotes any quantity satisfying $S(r, f)=o\{T(r, f)\}$ as $r \rightarrow+\infty$, possibly outside a set of $r$ of finite linear measure.

For completeness, we recall basic notions to this end: Given a meromorphic function $f$ and a constant $c, f(z+c)$ is called a shift of $f(z)$. For the sake of simplicity, we let $\Delta f(z)=f(z+1)-f(z), \Delta^{n} f(z)=\Delta\left(\Delta^{n-1} f(z)\right)(n \geq 2)$. As for a difference product, we mean a difference monomial of type $\prod_{j=1}^{k} f\left(z+c_{j}\right)^{n_{j}}$, where $c_{1}, \ldots, c_{k}$ are complex constants, and $n_{1}, \ldots, n_{k}$ are natural numbers.

Definition 1.1 A difference polynomial, resp. a differential-difference polynomial, in $f$ is a finite sum of difference products of $f$ and its shifts, resp. of products of $f$, derivatives of $f$ and of their shifts, with all the coefficients of these monomials being small functions of $f$.

Consider a transcendental meromorphic function $f$ and let

$$
P(z, f)=\sum_{l=1}^{n} a_{l}(z) \prod_{j=0}^{k}\left\{f^{(j)}\left(z+c_{l j}\right)\right\}^{n_{l j}},
$$

where $a_{l}(l=1,2, \ldots, n)$ are small functions of $f$, and $c_{l j}(l=1,2, \ldots, n ; j=0,1, \ldots, k)$ are complex constants, and $n_{l j}(l=1,2, \ldots, n ; j=0,1, \ldots, k)$ are natural numbers.

(c) 2015 Zhang et al.; licensee Springer. This article is distributed under the terms of the Creative Commons Attribution 4.0 International License (http://creativecommons.org/licenses/by/4.0/), which permits unrestricted use, distribution, and reproduction in any medium, provided you give appropriate credit to the original author(s) and the source, provide a link to the Creative Commons license, and indicate if changes were made. 
Definition 1.2 We define the total degree $d$ of $P(z, f)$ by

$$
d:=d(P(z, f))=\max _{1 \leq l \leq n}\left\{\sum_{j=0}^{k} n_{l j}\right\} .
$$

Moreover, a functional equation involving $f$ and its shifts as well as their derivatives is called a differential-difference equation.

Recently, many papers (see, e.g., [3] and [4]) are focused on complex difference polynomials and difference equations. Lots of results have been obtained by using value distribution theory (see, e.g., [5] and [6, 7]).

Yang and Laine [8] considered the following difference equation and proved the following.

Theorem A A non-linear difference equation

$$
f^{3}(z)+q(z) f(z+1)=c \sin b z
$$

where $q$ is a non-constant polynomial and $b, c \in \mathbb{C}$ are nonzero constants, does not admit entire solutions of finite order. If $q$ is a nonzero constant, then the above equation possesses three distinct entire solutions of finite order, provided that $b=3 n \pi$ and $q^{3}=(-1)^{n+1} c^{2} 27 / 4$ for a nonzero integer $n$.

Very recently, Liu et al. [9] proved the following.

Theorem B Let $n \geq 4$ be an integer, $q$ be a polynomial, and $p_{1}, p_{2}, \alpha_{1}, \alpha_{2}$ be nonzero constants such that $\alpha_{1} \neq \alpha_{2}$. Iff is an entire solution of finite order to the following equation:

$$
f^{n}(z)+q(z) \Delta f(z)=p_{1} e^{\alpha_{1} z}+p_{2} e^{\alpha_{2} z}
$$

then $q$ is a constant, and one of the following relations holds:

(1) $f(z)=c_{1} \exp \left(\frac{\alpha_{1}}{n} z\right)$, and $c_{1}\left(\exp \left(\alpha_{1} / n\right)-1\right) q=p_{2}, \alpha_{1}=n \alpha_{2}$;

(2) $f(z)=c_{2} \exp \left(\frac{\alpha_{2}}{n} z\right)$, and $c_{2}\left(\exp \left(\alpha_{2} / n\right)-1\right) q=p_{1}, \alpha_{2}=n \alpha_{1}$, where $c_{1}, c_{2}$ are constants satisfying $c_{1}^{n}=p_{1}, c_{2}^{n}=p_{2}$.

In this paper, we are going to discuss the case when $n=3$ in the above theorem and prove some results in Section 3.

\section{Preliminaries}

In order to prove our conclusions, we need some lemmas.

Lemma 2.1 ([8]) Let $f$ be a transcendental meromorphic solution of finite order $\rho$ of a difference equation of the form

$$
H(z, f) P(z, f)=Q(z, f)
$$

where $H(z, f), P(z, f), Q(z, f)$ are difference polynomials in $f$ such that the total degree of $H(z, f)$ in $f$ and its shifts is $n$, and that the corresponding total degree of $Q(z, f)$ is less or 
equal to $n$. If $H(z, f)$ contains just one term of maximal total degree, then for any small enough $\varepsilon>0$,

$$
m(r, P(z, f))=O\left(r^{\rho-1+\varepsilon}\right)+S(r, f) .
$$

The following result is a Clunie [10] type lemma for the difference-differential polynomials of a meromorphic function $f$. It can be proved by applying Lemma 2.1 and stated as follows.

Proposition $2.1([8,11])$ If in the above lemma, $H(z, f)=f^{n}, P(z, f)$, and $Q(z, f)$ are differential-difference polynomials in $f$, then $m(r, P(z, f))=S(r, f)$.

The following result is an analogue of a result due to Mohon'ko and Mohon'ko [12] for differential equations.

Lemma 2.2 ([4]) Let $f$ be a non-constant meromorphic solution with finite order of

$$
P(z, f)=0,
$$

where $P(z, f)$ is difference polynomial in $f$, and let $\delta<1$ and $\varepsilon>0$. If $P(z, a) \not \equiv 0$ for a slowly moving target function a, that is, $T(r, a)=S(r, f)$, then

$$
m\left(r, \frac{1}{f-a}\right)=o\left(\frac{T(r+|c|, f)^{1+\varepsilon}}{r^{\delta}}\right)+o(T(r, f))
$$

for all $r$ outside of a possible exceptional set with finite logarithmic measure.

Remark 2.1 By some further analysis on the proof of Theorem 2.4 in [6], we can obtain the conclusion of Lemma 2.2 when $P(z, f)$ is a differential-difference polynomial in $f$.

Lemma 2.3 ([13]) Assume that $c$ is a nonzero constant, $\alpha$ is a non-constant meromorphic function. Then the differential equation $f^{2}+\left(c f^{(n)}\right)^{2}=\alpha$ has no transcendental meromorphic solutions satisfying $T(r, \alpha)=S(r, f)$.

\section{Main results}

Now, we shall derive the following result similar to that of Theorems A and B, for extended differential-difference equations. First, we will prove the following result.

Theorem 3.1 Let $q$ be a polynomial, and $p_{1}, p_{2}, \alpha_{1}, \alpha_{2}$ be nonzero constants such that $\alpha_{1} \neq \alpha_{2}$. If $f$ is an entire solution of finite order to the following equation:

$$
f^{3}(z)+q(z) \Delta f(z)=p_{1} e^{\alpha_{1} z}+p_{2} e^{\alpha_{2} z}
$$

then $q$ is a constant, and one of the following relations holds:

(1) $T(r, f)=N_{1)}\left(r, \frac{1}{f}\right)+S(r, f)$;

(2) $f(z)=c_{1} \exp \left(\frac{\alpha_{1}}{3} z\right)$, and $c_{1}\left(\exp \left(\alpha_{1} / 3\right)-1\right) q=p_{2}, \alpha_{1}=3 \alpha_{2}$;

(3) $f(z)=c_{2} \exp \left(\frac{\alpha_{2}}{3} z\right)$, and $c_{2}\left(\exp \left(\alpha_{2} / 3\right)-1\right) q=p_{1}, \alpha_{2}=3 \alpha_{1}$, 
where $N_{1)}\left(r, \frac{1}{f}\right)$ denotes the counting function corresponding to simple zeros of $f$, and $c_{1}, c_{2}$ are constants satisfying $c_{1}^{3}=p_{1}, c_{2}^{3}=p_{2}$.

Proof of Theorem 3.1 Suppose that $f$ is a transcendental entire solution of finite order of (3.1). For the simplicity, we replace $f(z), f^{\prime}(z), \Delta f(z), q(z)$ by $f, f^{\prime}, \Delta f, q$. By differentiating both sides of (3.1), we have

$$
3 f^{2} f^{\prime}+(q \Delta f)^{\prime}=\alpha_{1} p_{1} e^{\alpha_{1} z}+\alpha_{2} p_{2} e^{\alpha_{2} z} .
$$

From (3.1) and (3.2), we obtain

$$
\begin{aligned}
& \alpha_{2} f^{3}+\alpha_{2} q \Delta f-3 f^{2} f^{\prime}-(q \Delta f)^{\prime}=\left(\alpha_{2}-\alpha_{1}\right) p_{1} e^{\alpha_{1} z}, \\
& \alpha_{1} f^{3}+\alpha_{1} q \Delta f-3 f^{2} f^{\prime}-(q \Delta f)^{\prime}=\left(\alpha_{1}-\alpha_{2}\right) p_{2} e^{\alpha_{2} z} .
\end{aligned}
$$

Differentiating (3.3) yields

$$
3 \alpha_{2} f^{2} f^{\prime}+\alpha_{2}(q \Delta f)^{\prime}-6 f\left(f^{\prime}\right)^{2}-3 f^{2} f^{\prime \prime}-(q \Delta f)^{\prime \prime}=\alpha_{1}\left(\alpha_{2}-\alpha_{1}\right) p_{1} e^{\alpha_{1} z} .
$$

It follows from (3.3) and (3.5) that

$$
f \varphi=T(z, f),
$$

where

$$
\begin{aligned}
& \varphi=\alpha_{1} \alpha_{2} f^{2}-3\left(\alpha_{1}+\alpha_{2}\right) f^{\prime} f+6\left(f^{\prime}\right)^{2}+3 f^{\prime \prime} f, \\
& T(z, f)=-\alpha_{1} \alpha_{2} q \Delta f+\left(\alpha_{1}+\alpha_{2}\right)(q \Delta f)^{\prime}-(q(z) \Delta f)^{\prime \prime} .
\end{aligned}
$$

Since $T(z, f)$ is a differential-difference polynomial in $f$ of degree 1 , it follows from (3.6) and Proposition 2.1 that $m(r, \varphi)=S(r, f)$, and $T(r, \varphi)=S(r, f)$. If $\varphi \not \equiv 0$, Lemma 2.2 gives $m\left(r, \frac{1}{f}\right)=S(r, f)$. This together with the first theorem will result in

$$
T(r, f)=N_{1)}\left(r, \frac{1}{f}\right)+S(r, f)
$$

where $N_{1)}\left(r, \frac{1}{f}\right)$ denotes the counting function corresponding to simple zeros of $f$, which is our desired result.

Next, we can assume that $\varphi$ in (3.6) vanishes identically, dividing with $f^{2}$, and recalling $f^{\prime \prime} \mid f=\left(f^{\prime} \mid f\right)^{\prime}+\left(f^{\prime} \mid f\right)^{2}$, we get the Riccati equation

$$
t^{\prime}+3 t^{2}-\left(\alpha_{1}+\alpha_{2}\right) t+\alpha_{1} \alpha_{2} / 3=0
$$

where $t:=f^{\prime} \mid f$. This equation has two constant solutions $t=\alpha_{1} / 3, t=\alpha_{2} / 3$. By Corollary 5.2 in the paper by Bank et al. [14], all other meromorphic solutions are of infinite order. Consider $t=\alpha_{1} / 3$, hence $f(z)=A \exp \left(\alpha_{1} z / 3\right)$ with a constant $A$ and look at (3.6). Denote $s(z):=q(z) \Delta f(z)$ and $u(z):=s^{\prime}(z) / s(z)$. Then, by (3.6), we get

$$
u^{\prime}=u^{2}+\left(\alpha_{1}+\alpha_{2}\right) u+\alpha_{1} \alpha_{2}
$$


As above, the only solutions of finite order are $u=\alpha_{1}, u=\alpha_{2}$, hence $s(z)=D_{1} \exp \left(\alpha_{1} z\right)$ or $s(z)=D_{2} \exp \left(\alpha_{2} z\right)$. Now, it is easy to see that the case $s(z)=D_{1} \exp \left(\alpha_{1} z\right)$ results in a contradiction. Therefore, we have $f(z)=A \exp \left(\alpha_{1} z / 3\right)$ and $q(z) \Delta f(z)=D_{2} \exp \left(\alpha_{2} z\right)$. The assertions $A\left(\exp \left(\alpha_{1} / 3\right)-1\right) q=p_{2}, A^{3}=p_{1}$ and $\alpha_{1}=3 \alpha_{2}$ now immediately follow, and the claim (2) is done. As to the claim (2), the Riccati equations for $t$ and $u$ are symmetric with respect to $\alpha_{1}, \alpha_{2}$, therefore the claim (3) follows.

This completes the proof of Theorem 3.1.

Remark 3.1 It is easy to see that $f(z)=e^{\pi i z}+e^{-\pi i z}=2 i \sin (\pi i z)$ is a solution of the following equation:

$$
f^{3}(z)+3 \Delta f(z)=e^{3 \pi i z}+e^{-3 \pi i z}
$$

Note $f(z)=e^{\pi i z}+e^{-\pi i z}=2 i \sin (\pi i z)$ has infinitely many zeros, and $T(r, f)=N_{1)}\left(r, \frac{1}{f}\right)+$ $S(r, f)$. Thus, we would like to pose the following conjecture.

Conjecture 3.1 If $\alpha_{1} \neq \alpha_{2}, \alpha_{1}+\alpha_{2} \neq 0$, then the conclusion (1) of Theorem 3.1 is impossible, in fact, any entire solution $f$ of (3.1) must have 0 as its Picard exceptional value.

For $\alpha_{1}+\alpha_{2}=0$, we have the following.

Theorem 3.2 Let $q_{j}(j=1,2,3)$ and $p$ be polynomials, and $\lambda_{1}, \lambda_{2}$, $\alpha$ be nonzero constants. Then non-linear difference equation

$$
f^{3}(z)+q_{3} \Delta^{3} f(z)+q_{2} \Delta^{2} f(z)+q_{1} \Delta f(z)=\lambda_{1} e^{\alpha z}+\lambda_{2} e^{-\alpha z}+p(z)
$$

possesses solutions of finite order of the form $f(z)=c_{1} e^{\alpha z / 3}+c_{2} e^{-\alpha z / 3}$ with $c_{1}^{3}=\lambda_{1}, c_{2}^{3}=\lambda_{2}$, and $p \equiv 0, v=e^{\alpha / 3}, q_{1}, q_{2}, q_{3}$ satisfy the condition:

$$
(v-1)^{3}\left(v^{3}+1\right) q_{3}+(v-1)^{2}\left(v^{3}-v\right) q_{2}+(v-1)\left(v^{3}+v^{2}\right) q_{1}=0 .
$$

Moreover, if $\alpha=(6 n \pi \pm 3 \pi) i$, then $\left(8 q_{3}-4 q_{2}+2 q_{1}\right)^{3}=27 \lambda_{1} \lambda_{2}$; or if $\alpha=(6 n \pi \pm \pi) i$, then $q_{2}+q_{1}=0$ and $\left( \pm \sqrt{3} i q_{1}-q_{3}\right)^{3}=27 \lambda_{1} \lambda_{2}$, where $n$ is an integer; if $v \neq-1, \frac{1 \pm \sqrt{3} i}{2}$, then $q_{1}, q_{2}$, $q_{3}$ satisfy the equation

$$
(v-1)^{2}\left(v^{2}-v+1\right) q_{3}+v(v-1)^{2} q_{2}+v^{2} q_{1}=0 .
$$

Proof of Theorem 3.2 For the simplicity the argument $z$ will be omitted in $f(z), f^{\prime}(z), \Delta f(z)$ etc. Suppose that $f$ is a transcendental entire solution of finite order of (3.8), and by differentiating (3.8) we get

$$
\begin{aligned}
& 3 f^{2} f^{\prime}+q_{3}^{\prime} \Delta^{3} f+q_{3}\left(\Delta^{3} f\right)^{\prime}+q_{2}^{\prime} \Delta^{2} f+q_{2}\left(\Delta^{2} f\right)^{\prime}+q_{1}^{\prime} \Delta f+q_{1}(\Delta f)^{\prime} \\
& \quad=\lambda_{1} \alpha e^{\alpha z}-\lambda_{2} \alpha e^{-\alpha z}+p^{\prime} .
\end{aligned}
$$

Combining (3.8) and (3.9), we obtain

$$
\begin{aligned}
& \alpha\left(f^{3}+q_{3} \Delta^{3} f+q_{2} \Delta^{2} f+q_{1} \Delta f\right)+3 f^{2} f^{\prime}+q_{3}^{\prime} \Delta^{3} f+q_{3}\left(\Delta^{3} f\right)^{\prime} \\
& \quad+q_{2}^{\prime} \Delta^{2} f+q_{2}\left(\Delta^{2} f\right)^{\prime}+q_{1}^{\prime} \Delta f+q_{1}(\Delta f)^{\prime}=2 \lambda_{1} \alpha e^{\alpha z}+\alpha p+p^{\prime}
\end{aligned}
$$


and

$$
\begin{aligned}
& \alpha\left(f^{3}+q_{3} \Delta^{3} f+q_{2} \Delta^{2} f+q_{1} \Delta f\right)-\left\{3 f^{2} f^{\prime}+q_{3}^{\prime} \Delta^{3} f+q_{3}\left(\Delta^{3} f\right)^{\prime}\right. \\
& \left.\quad+q_{2}^{\prime} \Delta^{2} f+q_{2}\left(\Delta^{2} f\right)^{\prime}+q_{1}^{\prime} \Delta f+q_{1}(\Delta f)^{\prime}\right\}=2 \lambda_{2} \alpha e^{-\alpha z}+\alpha p-p^{\prime},
\end{aligned}
$$

thus through the above two equations, we find

$$
f^{4}\left[-9\left(f^{\prime}\right)^{2}+\alpha^{2} f^{2}\right]=T_{4}(z, f)
$$

where $T_{4}(z, f)$ is a differential-difference polynomial of $f$, and its total degree is at most 4 .

If $T_{4}(z, f) \equiv 0$, it follows from (3.10) that $-9\left(f^{\prime}\right)^{2}+\alpha^{2} f^{2} \equiv 0$, then $f^{\prime}= \pm \frac{\alpha}{3} f$, and there exists a nonzero constant $c$ such that $f=c e^{ \pm(\alpha z) / 3}$. Substituting the above expression of $f$ into (3.8), we obtain a contradiction. Therefore, $T_{4}(f) \not \equiv 0$. Set $\beta=-9\left(f^{\prime}\right)^{2}+\alpha^{2} f^{2}$, then Proposition 2.1 shows that $m(r, \beta)=S(r, f)$, i.e. $\beta$ is a small function of $f$. Moreover, by Lemma 2.3 , it is easy to see that $\beta$ is a constant. By differentiating both sides of $\beta=-9\left(f^{\prime}\right)^{2}+$ $\alpha^{2} f^{2}$, we get

$$
f^{\prime \prime}-\left(\frac{\alpha}{3}\right)^{2} f=0
$$

It follows from (3.11) that

$$
f(z)=c_{1} e^{\alpha z / 3}+c_{2} e^{-\alpha z / 3}
$$

where $c_{1}, c_{2}$ are nonzero constants. Substituting (3.12) into (3.8), we have

$$
a_{1} w^{6}+a_{2} w^{4}+p w^{3}+a_{3} w^{2}+a_{4}=0
$$

where $w=e^{(\alpha z) / 3}, a_{1}=c_{1}^{3}-\lambda_{1}, a_{2}=3 c_{1}^{2} c_{2}+q_{3} c_{1} e^{\alpha}-3 q_{3} c_{1} e^{2 \alpha / 3}+3 q_{3} c_{1} e^{\alpha / 3}-c_{1} q_{3}+q_{2} c_{1} e^{2 \alpha / 3}-$ $2 q_{2} c_{1} e^{\alpha / 3}+q_{2} c_{1}+q_{1} c_{1} e^{\alpha / 3}-q_{1} c_{1}, a_{3}=3 c_{1} c_{2}^{2}+c_{2} q_{3} e^{-\alpha}-3 q_{3} c_{2} e^{-2 \alpha / 3}+3 q_{3} c_{2} e^{-\alpha / 3}-c_{2} q_{3}+$ $c_{2} q_{2} e^{-2 \alpha / 3}-2 c_{2} q_{2} e^{-\alpha / 3}+c_{2} q_{2}+c_{2} q_{1} e^{-\alpha / 3}-c_{2} q_{1}, a_{4}=c_{2}^{3}-\lambda_{2}$.

Since $w$ is transcendental, we must have $a_{1}=a_{4}=0$, and $a_{2}=a_{3}=p \equiv 0$. Therefore,

$$
\begin{aligned}
& q_{3}\left(e^{\alpha / 3}-1\right)^{3}+q_{2}\left(e^{\alpha / 3}-1\right)^{2}+q_{1}\left(e^{\alpha / 3}-1\right) \\
& \quad=q_{3}\left(e^{-\alpha / 3}-1\right)^{3}+q_{2}\left(e^{-\alpha / 3}-1\right)^{2}+q_{1}\left(e^{-\alpha / 3}-1\right) .
\end{aligned}
$$

Set $v=e^{\alpha / 3}$, it follows by (3.13) that

$$
q_{3}(v-1)^{3}+q_{2}(v-1)^{2}+q_{1}(v-1)=q_{3}\left(\frac{1}{v}-1\right)^{3}+q_{2}\left(\frac{1}{v}-1\right)^{2}+q_{1}\left(\frac{1}{v}-1\right)
$$

or

$$
\left(v^{2}-1\right)\left\{(v-1)^{2}\left(v^{2}-v+1\right) q_{3}+v(v-1)^{2} q_{2}+v^{2} q_{1}\right\}=0 .
$$

Now we distinguish four cases. 
Case 1. Assume that $v=1$, then $\alpha=6 n \pi i$, substituting $v=1$ into $a_{2}$ gives $c_{1} c_{2}=0$, this is a contradiction, thus $v \neq 1$.

Case 2. If $v=-1$, then $\alpha=(6 n \pi \pm 3 \pi) i$. By substituting $v=-1$ into $a_{2}$, we deduce $\left(8 q_{3}-\right.$ $\left.4 q_{2}+2 q_{1}\right)^{3}=27 \lambda_{1} \lambda_{2}$.

Case 3. While $v=\frac{1 \pm \sqrt{3} i}{2}$, in a similar way to above, we get $\alpha=(6 n \pi \pm \pi) i$ and $q_{1}+q_{2}=0$. Moreover, $\left( \pm \sqrt{3} i q_{1}-q_{3}\right)^{3}=27 \lambda_{1} \lambda_{2}$ and $\operatorname{deg} q_{1}=\operatorname{deg} q_{2}=\operatorname{deg} q_{3}$.

Case 4. When $v \neq-1, \frac{1 \pm \sqrt{3} i}{2}$, by (3.14), we could get the following equation:

$$
(v-1)^{2}\left(v^{2}-v+1\right) q_{3}+v(v-1)^{2} q_{2}+v^{2} q_{1}=0 .
$$

This also completes the proof of Theorem 3.2.

Theorem 3.3 Let $p_{j}(j=1,2)$ be polynomials, $\lambda_{1}, \lambda_{2}, \alpha$ be nonzero constants, $d_{1}, d_{2}$ be constants, and $k$ be a positive integer. Iff is an entire solution of finite order to the following non-linear differential-difference equation:

$$
f^{3}(z)+p_{1}(z) f^{(k)}\left(z+d_{1}\right)+p_{2}(z) f\left(z+d_{2}\right)=\lambda_{1} e^{\alpha z}+\lambda_{2} e^{-\alpha z}
$$

then $f(z)=c_{1} e^{\alpha z / 3}+c_{2} e^{-\alpha z / 3}, c_{1}^{3}=\lambda_{1}, c_{2}^{3}=\lambda_{2}$,

$$
3 c_{1} c_{2}+p_{1} e^{d_{1} \alpha / 3}(\alpha / 3)^{k}+p_{2} e^{d_{2} \alpha / 3} \equiv 0
$$

and

$$
3 c_{1} c_{2}+p_{1} e^{-d_{1} \alpha / 3}(-\alpha / 3)^{k}+p_{2} e^{-d_{2} \alpha / 3} \equiv 0
$$

Proof of Theorem 3.3 For simplicity the argument $z$ will be omitted in $f(z), f^{\prime}(z), f^{\prime}\left(z+d_{1}\right)$ etc. Suppose that $f$ is a transcendental entire solution of finite order of (3.15), by differentiating (3.15) we get

$$
3 f^{2} f^{\prime}+Q(z, f)=\lambda_{1} \alpha e^{\alpha z}-\lambda_{2} \alpha e^{-\alpha z}
$$

where $Q(z, f)=p_{1}^{\prime}(z) f^{(k)}\left(z+d_{1}\right)+p_{1}(z) f^{(k+1)}\left(z+d_{1}\right)+p_{2}^{\prime}(z) f\left(z+d_{2}\right)+p_{2}(z) f^{\prime}\left(z+d_{2}\right)$. Obviously, $Q(z, f)$ is a differential-difference polynomial of $f$, and its total degree is at most 1 .

Set $Q_{1}(z, f)=p_{1}(z) f^{(k)}\left(z+d_{1}\right)+p_{2}(z) f\left(z+d_{2}\right)$. It follows by (3.15) and (3.16) that

$$
\alpha f^{3}+3 f^{2} f^{\prime}+\alpha Q_{1}(z, f)+Q(z, f)=2 \lambda_{1} \alpha e^{\alpha z}
$$

and

$$
\alpha f^{3}-3 f^{2} f^{\prime}+\alpha Q_{1}(z, f)-Q(z, f)=2 \lambda_{2} \alpha e^{-\alpha z}
$$

Thus, for the above two equations, we can also get (3.10) and by a similar method to the one above, we have (3.12). Consequently

$$
f\left(z+d_{2}\right)=c_{1} e^{d_{2} \alpha / 3} e^{\alpha z / 3}+c_{2} e^{-d_{2} \alpha / 3} e^{-\alpha z / 3}
$$


and

$$
f^{(k)}\left(z+d_{1}\right)=c_{1}\left(\frac{\alpha}{3}\right)^{k} e^{d_{1} \alpha / 3} e^{\alpha z / 3}+c_{2}\left(-\frac{\alpha}{3}\right)^{k} e^{-d_{1} \alpha / 3} e^{-\alpha z / 3}
$$

In view of (3.15), (3.12), (3.17), and (3.18), we obtain $f(z)=c_{1} e^{\alpha z / 3}+c_{2} e^{-\alpha z / 3}$ with $c_{1}^{3}=\lambda_{1}$, $c_{2}^{3}=\lambda_{2}$. Moreover, we have

$$
3 c_{1} c_{2}+p_{1} e^{d_{1} \alpha / 3}(\alpha / 3)^{k}+p_{2} e^{d_{2} \alpha / 3} \equiv 0
$$

and

$$
3 c_{1} c_{2}+p_{1} e^{-d_{1} \alpha / 3}(-\alpha / 3)^{k}+p_{2} e^{-d_{2} \alpha / 3} \equiv 0
$$

Thus, we obtain the desired result and finish the proof of this theorem.

It is not difficult to see that a similar argument can be used to obtain the following result.

Theorem 3.4 Let $f$ be an entire solution of finite order to the following non-linear differential-difference equation:

$$
f^{3}(z)+P_{1}\left(z, \Delta f(z), \ldots, f^{\prime}(z), \ldots, f^{(k)}(z)\right)=\lambda_{1} e^{\alpha z}+\lambda_{2} e^{-\alpha z}
$$

here $P_{1}$ is a linear polynomial in $\Delta f(z), \ldots, f^{\prime}(z), \ldots, f^{(k)}(z)$, with polynomials as its coefficients, and $\lambda_{1}, \lambda_{2}, \alpha \in \mathbb{C}$ are nonzero constants. Then $f(z)=c_{1} e^{\alpha z / 3}+c_{2} e^{-\alpha z / 3}$ with $c_{1}^{3}=\lambda_{1}$, $c_{2}^{3}=\lambda_{2}$.

\section{Conclusion}

In studying differential equations in the complex plane $\mathbb{C}$, it is always an interesting and quite difficult problem to prove the existence or uniqueness of the entire or meromorphic solution of a given equation. There have been many studies and results obtained lately that relate to the existence or growth of entire or meromorphic solutions of various types of complex difference equations and difference-differential equations. By our methods, one can discuss the entire solutions for the algebraic difference equation and differencedifferential equation of the form

$$
f^{n}(z)+P_{l}\left(z, \Delta f(z), \ldots, f^{\prime}(z), \ldots, f^{(k)}(z)\right)=\lambda_{1}(z) e^{\alpha_{1}(z)}+\lambda_{2}(z) e^{\alpha_{2}(z)}
$$

here $P_{l}$ is a difference-differential polynomial in $\Delta f(z), \ldots, f^{\prime}(z), \ldots, f^{(k)}(z)$, with polynomials as its coefficients, and $\operatorname{deg}\left(P_{l}\right) \leq n-2, \lambda_{1}, \lambda_{2}, \alpha_{1}, \alpha_{2}$ are nonzero polynomials.

Competing interests

The authors declare that they have no competing interests.

Authors' contributions

All authors contributed in drafting this manuscript. All authors read and approved the final manuscript.

\section{Author details}

${ }^{1}$ College of Science, China University of Petroleum (East China), Qingdao, 266580, P.R. China. ${ }^{2}$ Department of Mathematics, Nanjing University, Nanjing, 210093, P.R. China. 


\section{Acknowledgements}

The authors would like to thank the referee for his/her several important suggestions and for pointing out some errors in our original manuscript. These comments greatly improved the readability of the paper. This work was supported by the research funds of China University of Petroleum (No. 14CX06085A), the Special Funds of the National Natural Science Foundation of China (No. 11426217) and the Fundamental Research Funds for the Central Universities (No. 14CX02012A).

Received: 30 December 2014 Accepted: 27 April 2015 Published online: 09 May 2015

\section{References}

1. Hayman, WK: Meromorphic Functions. Clarendon, Oxford (1964)

2. Yi, HX, Yang, CC: Uniqueness Theory of Meromorphic Functions. Science Press, Beijing (1995)

3. Chiang, YM, Feng, SJ: On the Nevanlinna characteristic of $f(z+\eta)$ and difference equations in the complex plane. Ramanujan J. 16, 105-129 (2008)

4. Halburd, RG, Korhonen, RJ: Difference analogue of the lemma on logarithmic derivative with applications to difference equations. J. Math. Anal. Appl. 314, 477-487 (2006)

5. Chen, ZX: Growth and zeros of meromorphic solution of some linear difference equations. J. Math. Anal. Appl. 373, 235-241 (2011)

6. Laine, I, Yang, CC: Clunie theorems for difference and q-difference polynomials. J. Lond. Math. Soc. 76, 556-566 (2007)

7. Laine, I, Yang, CC: Value distribution of difference polynomials. Proc. Jpn. Acad., Ser. A, Math. Sci. 83, 148-151 (2007)

8. Yang, CC, Laine, I: On analogies between nonlinear difference and differential equations. Proc. Jpn. Acad., Ser. A, Math. Sci. 86, 10-14 (2010)

9. Liu, NN, Lü, WR, Shen, TT, Yang, CC: Entire solutions of certain type of difference equations. J. Inequal. Appl. 2014, 63 (2014)

10. Clunie, J: On integral and meromorphic functions. J. Lond. Math. Soc. 37, 17-27 (1962)

11. Zhang, RR, Chen, ZX: Value distribution of difference polynomials of meromorphic functions. Sci. Sin., Math. 42, 1115-1130 (2012) (in Chinese)

12. Mohon'ko, AZ, Mohon'ko, VD: Estimates of the Nevanlinna characteristics of certain classes of meromorphic functions and their applications to differential equations. Sib. Mat. Zh. 15, 1305-1322 (1974) (in Russian)

13. Yang, CC, Li, P: On the transcendental solutions of a certain type of nonlinear differential equations. Arch. Math. 82, 442-448 (2004)

14. Bank, BS, Gundersen, GG, Laine, I: Meromorphic solutions of the Riccati differential equation. Ann. Acad. Sci. Fenn., Ser. A 1 Math. 6, 369-398 (1981)

\section{Submit your manuscript to a SpringerOpen ${ }^{\mathcal{O}}$ journal and benefit from:}

- Convenient online submission

- Rigorous peer review

Immediate publication on acceptance

- Open access: articles freely available online

- High visibility within the field

- Retaining the copyright to your article 